\title{
The Challenges in the Fight against COVID-19 Pandemic: A Qualitative Study
}

\author{
Sanni Shamsudeen Ademola'), Neema Rajabu²), Aliyu Olugbenga Yusuf3) \\ 1)Department of Computer Science, Faculty of Science and Engineering, University of Eswatini \\ 2) Faculty of Computer Science and Information Technology, \\ Kampala International University, Dar es Salaam Campus, Tanzania \\ 3)Department of Library and Information Science, Federal University of Lafia, Nigeria
}

Background: COVID-19 pandemic has dominated the global space for more than a year, and the world faces a lot of challenges in the battle against the deadly virus. This study highlights fundamental issues that undermine the fight against the COVID-19pandemic and informs on how government and health authorities might further the cause of pandemic control. This study aimed is to analyze challenges that hinder the battle against the COVID-19pandemic from the viewpoint of the Nigerian Twitter community.

Sulbjects and Method: This was a qualitative study with phenomenological approach, conducted using an online survey through Google Forms with Nigerian Twitter community. A total of 128 informants were selected using a list-based sampling technique. The data was managed, cleaned, organized and analyzed in SPSS, Microsoft Excel Spreadsheet, Microsoft Word and Microsoft Notepad.

Results: $40.6 \%$ of the informants are in the field of Education, $11.7 \%$ are Entrepreneurs, $10.9 \%$ are Students, $10.9 \%$ are Civil Servants, 9.4\% are IT Specialists, while 7.0\% are Engineers. Issues that undermines the fight against COVID-19pandemic according to the findings from this study were summarized and classified into five themes, included: 1) Fake News Distort Health Messages on Covid-19, 2) Conspiracy Theories on Covid-19, 3) Mismanagement of COVID-19pandemic, 4) Lack of Government Trust on Covid-19, 5) Lack of Adequate Information on COVID-19.

Conclusion: Findings suggest that these five issues are key in fighting the COVID-19scourge and must be addressed in future pandemic mitigation efforts and control plans.

Keywords: COVID-19, health communication, fake news, pandemic, health messages

\section{Correspondence:}

Sanni Shamsudeen Ademola. Department of Computer Science, Faculty of Science and Engineering, University of Eswatini. Private Bag 4, Matsapha Manzini, The Kingdom of Eswatini. Email: sanniade01@gmail.com. Mobile: +26876241155/ 79241155

Cite this as:

Ademola SS, Rajabu N, Yusuf AO (2021). The Challenges in the Fight against COVID-19 Pandemic: A Qualitative Study. J Health Promot Behav. 06(02): 133-143. https://doi.org/10.26911/thejhpb.2021.06.02.06.

cc (i) (2) Journal of Health Promotion and Behavioris licensed under a Creative Commons

Attribution-NonCommercial-ShareAlike 4.o International License.

\section{BACKGROUND}

Several challenges are encountered in the fight against the COVID-19pandemic. There were well-reported challenges with enforcing regulations (Anwar et al., 2020; Caetano et al., 2020; Yezli and Khan, 2020), challenges with administering treatments (Caetano et al., 2020; Gopalan and Misra, 2020; Medeiros, 2020; Spinelli and Pellino, 2020; Wollina, 2020), challenges with vaccinations (Forni et al., 2021; Wouters et al., 2021) and so many other challenges. 
The information serves as a guide for actions and health information communication is a fundamental aspect of healthcare. Government and health autho $\neg$ rities prepare and announce measures, activities and habits to be practiced regularly to curb the spread of COVID-19. Such measures include wearing of face masks, hand sanitizing, social distancing, restrictions in any forms of gatherings, restrictions in movement, lockdown, working from home and virtual meetings. This was followed by mass testing, treatments and vaccination. All these measures were put in place and enforced to curb the spread of the disease. The world has made lots of progress in slowing down the spread of COVID-19 through both therapeutic and nontherapeutic preventive measures. However, general public opinion suggests that individuals' perceptions and convictions on COVID-19 contribute largely to influence their attitudes, behavior and decision making on COVID-19. In essence, this study attempts to uncover trends in thoughts, opinions and views from the Nigerian Twitter community, on the main issues of concern for them in the fight against the COVID-19pandemic.

This study draws upon behavioral theories to analyze an individual's decisionmaking, attitude and beliefs during the COVID-19 pandemic. These theories are suited to predicting behavior and are widely used on topics related to health. A useful theoretical model applicable to guide health promotion campaigns and disease prevention programs is the Health Belief Model (Rosenstock, 1974; Strecher and Rosenstock., 1997) which states that behavior is determined by several beliefs about threats to an individual's well-being and the effecttiveness and outcomes of particular actions or behaviors. These beliefs include perceived susceptibility, perceived severity, perceived benefits, perceived barriers, cues to action, and self-efficacy. Perceived susceptibility refers to an individual's perceived threat to sickness or disease which determines readiness to perform an action or change behavior. Perceived severity refers to an individual's belief of consequences that may result from sickness or disease which determines readiness to take action or alter behavior.

In the context of the COVID-19 pandemic, perceived susceptibility assumes that people are likely to change their behavior and follow COVID-19 regulations only if they believe they could become infected, sick or killed by the disease. Perceived severity assumes that it will be unlikely for people to change behavior and follow COVID-19 regulations if they are skeptical about the existence of COVID-19or curious about the figures of casualties being rolled out. Perceived benefits posit that it will be unlikely to observe any changes in behavior and attitude among people if they are not certain that following COVID-19 regulations will prevent them from getting infected or sick. Perceived barriers are of the assumptions that behavior change might be difficult if there are financial cost and negative consequences associated with following regulations. The perceived barriers with COVID-19regulations are cost of personal protective equipment (PPE), face Masks, Hand Sanitizers etc. This is in addition to negative consequences such as side effects of COVID-19treatments and vaccination. The model describes internal and external cues to action: internal includes symptoms of ill health, and external includes media campaigns and health communications through varieties of medium.

Another widely applied theory in behavior study is the Theory of Planned Behaviour and Theory of Reasoned Action (Fishbein, 1979; Ajzen, 1991). This followed a cognitive approach to explaining behavior 
with a focus on individual attitudes and beliefs. One key aspect of this theory is subjective norms, which involve social pressures that influence behavior and an individual's inclination to conform to social pressure. With regards to COVID-19 pandemic, social pressure may be experienced through family, friends, colleagues, and social media groups. Behaviour is influenced by its consequences much of the time, that is people choose to behave in certain ways knowing the consequences of their behavior. Many of the things we do are designed to gain anticipated benefits and to avert future trouble. It thus suggests that convictions about the COVID-19 pandemic play a large role in how individuals and communities manage the outbreak.

Therefore, this study aimed to collect and analyze information from the Nigerian Twitter community on what they perceive as the main challenges in the fight against COVID-19 pandemic.

\section{SUBJECTS AND METHOD}

\section{Study Design}

This study uses qualitative research with phenomenological approach. Research design of inquiry which sought to describe lived experiences of study participants in the COVID-19era as described by the participants themselves. This involves conducting interviews that rely mainly on textual data. This method was adopted to be able to explore the research objectives in detail and uncover trends in thoughts, opinions and views on issues that affect the fight against COVID-19 pandemic in different communities.

An online survey amongst Nigerian Twitter community was conducted and the strategy of enquiry was a cross-sectional study through the use of structured online interview tool that leads to both numerical and textual analysis and provides a narra- tive description of perceptions, beliefs, and attitudes of the respondents during the COVID-19 pandemic. Qualitative research focuses on human behavior from a participant's point of view, as such the report of our findings is narrative and explanatory. An online survey amongst Nigerian Twitter community was conducted and the strategy of enquiry was a cross-sectional study through the use of structured online interview tool that leads to both numerical and textual analysis and provides a narrative description of perceptions, beliefs, and attitudes of the respon-dents du $\neg$ ring the COVID-19 pandemic.

Data collection procedure was through a self-administered online structured interview designed in Google Forms. The survey was cross-sectional, with the data collected at one point in time. Data was collected through a structured online survey designed through free Google Forms application. designed through free Google Forms application. We initially conducted a pilot test of the interview questions to check for errors and to be sure that the questions being asked are understood the same way across board and to avoid any ambiguity. The link to the survey page was sent to participants' Twitter inbox. This is to be certain that participants are individuals with the ability to use online platforms for information access and interaction. To administer the survey, the link was sent out to participants with information on the purpose of the research and instructions on how to answer the questions. We sent a reminder a week after the first inbox was sent.

The data was collected from December 27th, 2020 to January 26th, 2021 (One month period).

\section{Study Informants}

The collection of informants in this study uses purposive sampling, which is the 
technique of determining samples based on researchers knowledge about the study and population. Nigeria has the highest percentage of Twitter users in Africa and amongst the top countries shaping African Twitter conver $\neg$ sations on COVID-19. The targeted population were deemed to be computer literates and have both regular and easy access to the internet to facilitate responding to the survey. Besides, they were deemed to be knowledgeable enough to provide answers to the questions surround $\neg$ ing COVID-19pandemic since it is a global pandemic experienced by everyone. A singlestage sampling procedure was followed in which the researchers have access to Twitter accounts of potential respondents which is the technique of determining samples with certain considerations or criteria:

1) Esers of Twitter microblogging in Nigeria

2) individuals who tweets or comments on tweets related to Covid-19

\section{Data Analysis}

The purpose of the data analysis is to make sense out of the textual data that was collected. Data was organized and prepared for analysis in SPSS, Microsoft Excel Spreadsheet, Microsoft Word and Microsoft Notepad. These data analysis software was logically helpful in organizing, sorting, and searching for information in the text transcripts. The data was cleaned to obtain a broader sense of the information. We relied on both inductive and deductive data analysis by identifying keywords, categories, and building patterns and themes from the textual data.

The data collected went through a reliability and validation process to avoid mistakes and errors in the coding process. The text transcripts were given to 3 English language experts to read, interpret and assign codes. The code assignments from these exercises were cross-checked for relia- bility and validity across board to ascertain internal consistency and to achieve intercoder agreement. During this process, some codes were merged, some were dropped for failing the validity text, while some were dropped for lack of clarity. Data was represented in the form of tables, graphs and in narrative forms. The data was later hand-coded to form a description, extract keywords, identify and group them into themes. In the process of hand-coding, we read through each line of text to get a general sense and reflect on its overall meaning in order to be able to accurately assign codes. Themes that were similar or related were grouped together to demonstrate a higher level of analysis and abstractions.

The keywords and themes were identified and grouped based on the objectives of the study. This reflects on the overall depth, credibility, and usefulness of the information obtained. In reporting the findings from the analysis of the data, we applied the 'winnowing' approach by focusing in on some of the data and disregarding some other parts of it (especially those where different respondents make similar statements or reported similar events). The codes that were identified in this study were developed on the basis of the emerging information collected from participants, that is, the codes emerged during the data analysis and were not predetermined. The dependent variable was worship behavior.

\section{RESULTS \\ Sociodemographic characteristic of participants}

This study collected information from 128 informants, with mean age of 36.87 years, with minimum age 17 and maximum age 78 The characteristics of study informants (age) is represented in Table 1. 
Ademola et al./ The Challenges in the Fight against COVID-19 Pandemic

Table 1. The Characteristics of Study Informants (Age)

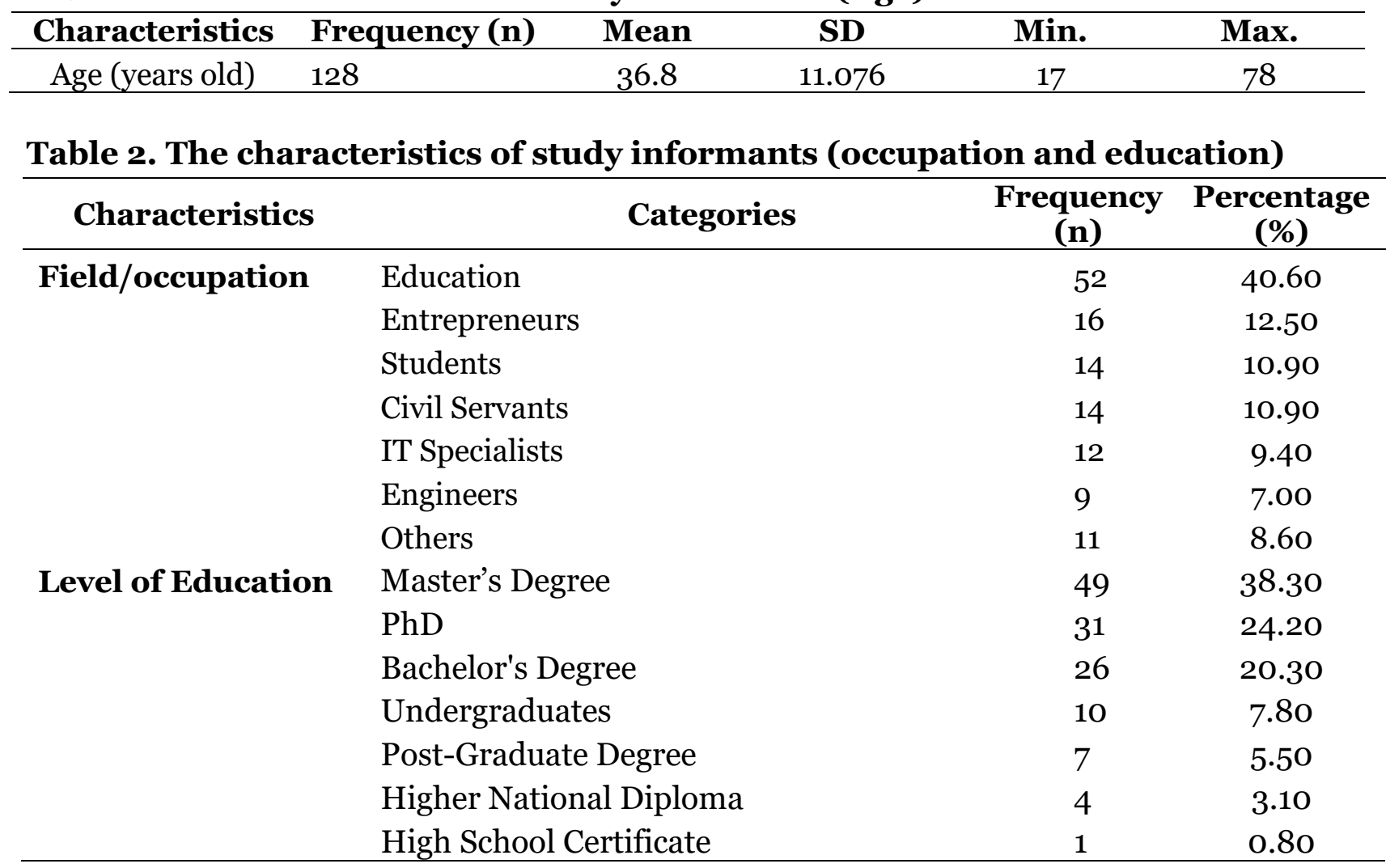

Table 2 showed that majority (40.6\%) are in the field of education, University lecturers, researchers and teachers, followed by entrepreneurs (11.7\%), students (10.9\%), Civil Servants (10.9\%), IT Specialists (9.4\%), and engineers (7.0\%). The level of education of study informants suggests that the majority of them are highly educated individuals with $24.2 \%$ of them with PhDs. Majority of them possess master's degree certificates (38.3\%), followed by $\mathrm{PhDs}$ (24.2\%), bachelor's degrees (20.3\%), undergraduates $(7.8 \%)$, post-graduate degrees (5.5\%), higher national diploma (3.1\%), high school sertificate (o.8\%).

Challenges in the Fight against COVID-19pandemic

This study provides insights into the perception of the Nigerian Twitter community on the key issues that hinders the fight against COVID-19pandemic. The 5 main themes that manifest as the biggest challenges during the pandemic in their order of great concerns are as follows: 1) Fake News Distort Health Messages on Covid-19, 2) Conspiracy Theories on Covid19, 3) Mismanagement of COVID-19pandemic, 4) Lack of Government Trust on COVID-19, 5) Lack of Adequate Information on COVID-19.

\section{1) Fake news distort health messages on COVID-19}

The theme that was more manifest in the data analysis was the fact that fake news distorts health messages on Covid-19, suggesting that the biggest challenge faced during COVID-19pandemic is fake news and misinformation, especially fake news posted and shared through social media.

"People on social media more especially on Facebook, WhatsApp create fake information about COVID-19 and share it"

"Fake news is highly comercialized this time specially for global issues like COVID-19, so I personally feel 
Ademola et al./ The Challenges in the Fight against COVID-19 Pandemic

the media owners should seriously evaluate their media regularly and filter malicious information since the issue is serious"

As much as there exist large amounts of fake news spreading all over social media, useful information on COVID-19 is also shared through social media platforms.

"Social media has a serious problem with regards to information on COVID 19. Some of information on social media was helpful at times, and it has likewise caused harm sometimes"

It is glaring that fake news distorts health messages during the COVID-19 pandemic and we would need to combat fake news in order to control any pandemic. Hence, we must place significant emphasis on controlling fake news and misinformation.

\section{2) Conspiracy Theories on COVID-19}

Amongst the participants of this study are also those who believed Covid-19 is a big lie and are of the opinion that there is a big conspiracy surrounding the whole pandemic. Some of their views are shared below:

"Many people in my country believe COVID-19 is a lie, a propaganda, but I believe it exist but there are several lies around it"

"The Western giants should focus Covid-19 related attention on the more affected region, and stop unnecessary brouhaha about Africa"

There is also a viewpoint that the general public do not really know the truth about the disease.

"I still believe there is an untold story about the origin of the disease"

"I feel nobody knows the true picture behind COVID-19 except the almighty God"
Some participants are of the opinion that COVID-19 was created to control the world population and world economy.

"At first, they made us believe the pandemic was deadly, hence locking us down, crippled businesses and livelihoods of the many. It's much clearer now that the whole COVID brouhaha was indeed a plandemic not pandemic as it were"

"Politicians around the globe and globalist elites want to control people's lives, thereby taking us back to the communist era. People are more aware now more than ever before. Their schemes and gimmicks have expiry dates"

"COVID-19 is a grand scheme of the conspiracy theorists to manipulate and mislead the human race for reasons best known to them (the theories)"

"My general thought about the Covid-19 is just like the world is coming to an end, as most leaders were not faithful to their followers and we are just being deceived about a lot of things about the virus and looking for ways to enslave everyone"

These excerpts reflect that one of the major challenges to fighting COVID-19 pandemic is conspiracy theories. It reveals that there is quite a large percentage of people around the globe who are skeptical about the existence and spread of COVID-19. This suggests that one of the issues that must be properly addressed during a pandemic is conspiracy theories.

\section{3) Mismanagement of COVID-19 pan- demic}

According to findings from this study, one of the main issues that hinders the control of the pandemic is mismanagement from the authorities. There is a belief that the 
pandemic was not properly managed by those put in charge.

"A Lot of contradictory information is coming from the authority and this affect people a lot"

"Governments and the health specialists took so long to inform citizens on how to prevent COVID-19 infections and what to use to boost one's immune system which I found disturbing"

Another important issue raised by the informants is the inconsistencies and confusions in Covid-19 regulations. To some of the participants, this really affected behavior and raised suspicions about the severity of the pandemic.

'The measure put in place for testing does not allow people to believe the disease is serious. The guidelines and measures put in place to control the second wave does not address the present situation. Currently, the international airports are open and people are trooping in from different countries. To me, we are not fighting $d$ pandemic as it should be"

"It is hilarious and ridiculous to see different rules unfold at various times, countering opinions from same government agencies as to how the disease is passed from one person to another"

Similarly, the information being disseminated on the science of the disease by the government became suspect. People receive contradictory information on the science of the disease through government portals, government agencies and health authorities on COVID-19.

"Many of the information from healthcare workers and first responders are misleading and confusing"
"People in government just send forth any information they received without verifying if it is wrong or correct and sadly, we later found that those information were not accurate"

"A lot of information that I thought was true were later confirmed to be mere political statements and conspiracy theories so much so that it is difficult to judge whatever one hears, reads or sees"

These revelations were supported by previous researchers who observed that during disease outbreaks, there were reported cases where caregivers and essential service providers also demonstrate lack of proper knowledge of the disease and this terribly hinders control and mitigation efforts (Maunder et al., 2003; Perrett et al., 2003; Lin CY, 2020).

\section{4) Lack of Government Trust}

One of the major issues that hinders the fight against COVID-19 pandemic is lack of government trust. This is according to findings from this study.

"The government is actually trying but the citizens do not really trust or believe the government"

"NCDC, CDC and WHO share Facts about COVID-19. The Question now is if I trust those facts. My reality seems extremely different from the Facts / Fears shared. Reality is that COVID-19 ought to be demystified and not feared and that the human relationships or economy can return to normal whilst continous measures are put in place to limit spread and enable speedy harmless recoveries"

Some participants are worried that the government and politicians are taking advantage of the COVID-19 situations for financial gains.

"The greatest problem is that most politicians are taking advantage of 
Ademola et al./ The Challenges in the Fight against COVID-19 Pandemic

Covid-19 for personal gains and this is affecting the proper management of the disease"

"It is also true that economies have been unnecessarily hit hard by the uncalled-for lockdowns just for Governments to make billions out of the situation"

"Most people in my country do not believe that Covid-19 exists. But personally, I know it exists, although it's not severe in my country. However, the Government is using it as an avenue to steal public funds"

Some participants question the infection figures released daily by the government on COVID-19. They cited lack of transparency, honesty and openness by their government, especially with Covid-19 statistics.

"Government misled its citizens with the news about the spread of COVID19 within its borders. They deliberately inflate the number of cases to create fear"

"As much as I believe Covid-19 exists, I strongly believe the figures being released by NCDC are being inflated due to greed"

"I believe the figures provided by the government to the media each day are not true and it becomes difficult to use it for true assessment of the COVID 19 pandemic in our environment"

\section{5) Lack of Adequate Information}

When there is no availability of proper, adequate information, we give rooms for rumour mongering. To some participants, the lack of adequate information prevents people from adhering to regulations and taking proper precautions.

"More information should be shared regarding prevention of COVID-19, to confirm how reliable is COVID-19 vaccine and its side effects"

"Misinformation increases vulnerability of the poor people in rural areas who do not have adequate sources of receiving information and who do not have capacity to verify genuine or fake information"

"What are the strategies to curb misinformation from family members or friends who do not have means of listening to news, radio or social media?"

It is obvious from the above responses that lack of information penetration also hinders the fight against COVID-19 pandemic. In essence, adequate and far-reaching information is vital to fighting a pandemic.

\section{DISCUSSION}

This study has highlighted fundamental issues that create obstacles in the fight against COVID-19. The result of our findings suggests that to fight the COVID-19 pandemic or any disease outbreak for that matter, government, health authorities, organizations and leading voices in health campaigns must address a) Fake News and misinformation, b) Conspiracy theories, c) Management of the Crises, d) Government Trust, e) Adequate Information on the Crisis.

The topmost issue that is of great concern during the COVID-19 pandemic is that fake news and misinformation distort health messages on COVID-19. Infodemic was apparent, which according to the World Health Organization is an overdose of false and misleading information during a disease outbreak. Infodemic leads to confusion, fear, anxiety and risk-taking behaviors that affect healthcare. It also leads to mistrust in government and health authorities and undermines the public health response. Similarly, conspiracy theories on COVID-19 also 
undermine mitigation and control efforts. We found that many people are skeptical about the origin, existence and spread of the disease. This results in mistrust in health authorities and health messages. Therefore, in order to stem the tide of disease outbreak, conspiracy mongers must be put to check through proper, adequate and direct health messages that are far reaching.

Another issue of concern to the general public is how the COVID-19 crisis was mismanaged in many countries and this discouraged lots of people from complying with regulations. Information about the pandemic was not properly managed, measures and regulations were not well managed, treatments and interventions were mismanaged and we can now see that the vaccination process has also been mismanaged in many countries. Thus, controlling an epidemic demand for proper management of information, interventions, and treatments, as this will boost the confidence of the masses in the process. Our findings also revealed that lots of people have trust issues with their government and politicians, and it becomes very hard to believe anything or any claim the government makes on COVID-19. The popular sentiment is that the government and politicians are corrupt and cannot be trusted with information. The perception is that the government politicizes the pandemic for financial, material and political gains. Participants lament the indiscipline and insincerity on the part of the government when it comes to following regulations, since most government officials and politicians have no restrictions in movement, gatherings and socializing in the middle of the pandemic. This behavior has a negative effect on the psychology of the general population with regards to obeying Covid19 rules and regulation. Therefore, in order to successfully control the spread of a pandemic, the government and politicians must be seen to lead in practicing and following all laid down regulations.

Besides, we found that lack of adequate information frustrates mitigation and control measures. When there is a lack of adequate and reliable information, we give rooms for doubts, conjectures, confusion and risk-taking behaviours. Lack of proper information also leads to self-medication and practices that could be injurious to health. In essence, adequate, deliberate, reliable and focused information is crucial to fighting a pandemic. Misinformation counteracted mitigation and control efforts, negatively affecting interventions and good health behaviour. Apparently, the world is not new to misinformation during disease outbreak, infodemic was a serious problem in 2003 during the outbreak of SARS, in 2014 during the Ebola disease outbreak (Maunder et al., 2003; Cheung, 2015) and 2016 during the Zika virus outbreak (Ghenai and Mejova, 2017; Bode and Vraga, 2018). Misinformation also resulted in hostilities towards aid workers and healthcare professionals during the Covid-19 pandemic, similar occurrence was reported in Liberia during Ebola outbreak (Cheung, 2015).

There is already a burden of fear and anxiety on healthcare workers with the additional stress that comes with intimidation, all resulting from misinformation. The fact that misinformation was spreading unabated, and negatively affected mitigation efforts during COVID-19 pandemic indicated that we need to extend mitigation efforts beyond treatments and medical interventions and expand it to the media spaces. The media narratives on COVID-19 pandemic present sometimes accurate, and other times inaccurate information on the pandemic. We have experienced news out- 
lets contradict one another in their news bulletins, and oftentimes come out to retract earlier statements or make clarifications on previously broadcasted news. As the COVID-19 pandemic has shown, contradictions from health messages negatively affect disease control. Findings and discussion from this study was based on individuals on Twitter microblogging platform who are generally young, working class and educated. Future researchers could seek opinions of individuals from other social systems or demographics in order to expand our stock of knowledge on the subject matter. We encourage government and health authorities to consider and include our findings in future pandemic control efforts and plans.

\section{AUTHOR CONTRIBUTION}

Sanni Shamsudeen Ademola concepttualized and designed the research idea, he coordinated the study and was involved in co-writing the entire paper. Neema Rajabu was actively involved in designing the research framework and data collection tools and likewise in co-writing the entire paper. Aliyu Olugbenga Yusuf was actively involved in conducting literature review for the study, participated in the data collection, data processing, data analysis and interpretation of results.

\section{FUNDING AND SPONSORSHIP}

This study is self-sponsored.

\section{CONFLICT OF INTEREST}

There is no conflict of interest in this study.

\section{ACKNOWLEDGMENT}

The researchers are grateful to individuals who took their time to participate in this study and we also acknowledge Google and Twitter for allowing us to use their platforms for free to conduct the research.

\section{REFERENCES}

Ajzen I, (1991). The theory of planned behavior. Organizational Behavior and Human Decision Processes.; 5O(2): 179-211. DOI: 10.1016/07495978(91)90020-T

Anwar S, Nasrullah M, Hosen MJ (2020). Covid-19 and bangladesh: challenges and how to address them. Frontiers in Public Health. 8: 154. DOI: 10.3389/fpubh.2020.00154.

Bode L, Vraga EK (2018). See something, say something: correction of global health misinformation on social media. Health Communication. Routledge. 33(9): 1131-1140. DOI:10.1080/10410236.2017.1331312.

Caetano R, Silva AB, Guedes ACCM, Paiva $\mathrm{CCN}$, Riberio GdR, Santos DL, et al., (2020). Challenges and opportunities for telehealth during the COVID-19 pandemic: Ideas on spaces and initiatives in the Brazilian context. Cadernos de Saude Publica.; 36(5): pp. 116. DOI:10.1590/0102311Xooo88920

Cheung EYL (2015). An outbreak of fear, rumours and stigma. Intervention.; 13(1): 70-76. DOI: 10.1097/wtf.oooooooooooooo79.

Fishbein MA, (1980). Theory of reasoned action: some applications and implications. Nebr Symp Motiv. 27:65-116. PMID: 7242751.

Forni G, Mantovani A (2021). COVID-19 vaccines: where we stand and challenges ahead. Cell Death and Differentiation. 28(2): 626-639. DOI: 10.1038/s41418-020-00720-9.

Ghenai A, Mejova Y (2017). Catching zika fever: application of crowdsourcing and machine learning for tracking health misinformation on twitter. Proceeding -2017 IEEE International Conference on Healthcare Informatics, ICHI 2017. p. 518. DOI: 10.1109/- 
Ademola et al./ The Challenges in the Fight against COVID-19 Pandemic

ICHI.2017.58.

Gopalan HS, Misra A (2020). COVID-19 pandemic and challenges for socioeconomic issues, healthcare and National Health Programs in India. Diabetes \& Metabolic Syndrome: Clinical Research \& Reviews. 14(5): 757-759.

Lin CY (2020). Social reaction toward the 2019 Novel Coronavirus (COVID-19). Soc Health Behav. 3(1-2): 20192020. DOI: 10.4103/SHB.SHB_11_20

Maunder R, Hunter J, Vincent L, Bennett J, Peladeau N, Leszcz M, Sadavoy J, Verhaeghe, LM, Steinberg R, Mazzulli $\mathrm{T}$ (2003). The immediate psychological and occupational impact of the 2003 SARS outbreak in a teaching hospital. Cmaj. 168(10): 1245-1251.

Medeiros EA S (2020). Challenges in the fight against the COVID-19 pandemic in university hospitals. Revista Paulista de Pediatria. 38. DOI: 10.1590/19840462/2020/38/2020086.

Perrett K, al-Wali W, Read C, Redgrave P, Trend U (2000). Outbreak of meningococcal disease in Rotherham illustrates the value of coordination, communication, and collaboration in management. Commun Dis Public Health. (3): 168-71. PMID: 11014027.

Rosenstock IM (1974). The health belief model and preventive health behavior. Health education monographs. 2(4): 354-386. http://dx.doi.org/10.1177/109019817400200407.

Spinelli A, Pellino G (2020). COVID-19 pandemic: perspectives on an unfolding crisis. British Journal of Surgery. 107(7): 785-787. DOI: 10.1002/bjs.11627.

Strecher VJ, Rosenstock IM (1997). The health belief model. Cambridge handbook of psychology, health and medicine. Cambridge: 117. DOI: 10.1017/CBO9780511543579.

Wollina U (2020). Challenges of COVID-19 pandemic for dermatology. Dermatologic Therapy. 33(5): 1-5. DOI: 10.1111/dth.13430.

Wouters OJ (2021). Challenges in ensuring global access to COVID-19 vaccines: production, affordability, allocation, and deployment. The Lancet. 397(10278): 1023-1034. DOI: 10.1016/So140-6736(21)00306-8.

Yezli S, Khan A (2020). COVID-19 social distancing in the Kingdom of Saudi Arabia: Bold measures in the face of political, economic, social and religious challenges. Travel Med Infect Dis. 37(101692): 1-4. DOI: 10.1016/j.tmaid.2020.101692. 